\title{
A Novel Saliency-Based Graph Learning Framework with Application to CBIR
}

\author{
Hong $\mathrm{BAO}^{\dagger \mathrm{a})}$, Song-He FENG ${ }^{\dagger \mathrm{b})}$, De $\mathrm{XU}^{\dagger}$, Nonmembers, and Shuoyan $\mathrm{LIU}^{\dagger}$, Student Member
}

\begin{abstract}
SUMMARY Localized content-based image retrieval (LCBIR) has emerged as a hot topic more recently because in the scenario of CBIR, the user is interested in a portion of the image and the rest of the image is irrelevant. In this paper, we propose a novel region-level relevance feedback method to solve the LCBIR problem. Firstly, the visual attention model is employed to measure the regional saliency of each image in the feedback image set provided by the user. Secondly, the regions in the image set are constructed to form an affinity matrix and a novel propagation energy function is defined which takes both low-level visual features and regional significance into consideration. After the iteration, regions in the positive images with high confident scores are selected as the candidate query set to conduct the next-round retrieval task until the retrieval results are satisfactory. Experimental results conducted on the SIVAL dataset demonstrate the effectiveness of the proposed approach.

key words: localized CBIR, graph learning, visual attention, relevance feedback
\end{abstract}

\section{Introduction}

With the increased number of digital images available on the Internet, efficient indexing and searching becomes an essential issue for large image archives. Content-based image retrieval (CBIR) has been widely developed in the last decade [1]. Most CBIR systems adopt the "query-byexample" paradigm, i.e., the user is asked to submit a query image to the system in order to retrieve similar images. Usually, the user targets one or several important objects in the query image only and does not care about the background. Earlier work on CBIR tried to use global low-level visual features to fulfill the retrieval task. Since the information of the interesting objects may be concealed by the background using global features, the retrieval results are very unsatisfying. One possibility to extract those interesting objects is using region-based approaches. Image segmentation technique is usually utilized to segment the image into regions with similar local visual features. As images often consist of different numbers of regions, how to solve the many-tomany matching problem becomes a prerequisite for measuring image similarity at the region level [6]. Since the user typically does not provide any indication of which portion of the image is of interest, identifying important regions in an image is still an open problem. Relevance feedback mechanism provides another solution to narrow down the semantic

Manuscript received October 19, 2010.

Manuscript revised February 20, 2011.

${ }^{\dagger}$ The authors are with the Institute of Computer \& Engineering, Beijing Jiaotong University, Beijing, China.

a)E-mail: xxtbaohong@buu.edu.cn (Corresponding author)

b)E-mail: shfeng@bjtu.edu.cn

DOI: $10.1587 /$ transinf.E94.D.1353 gap via user interaction. It is an interactive process by which the user marks the images retrieved by the system as relevant or irrelevant. According to user's feedback, either the matching criterion or the ideal query may be updated which subsequently improves the retrieval performance. More recently, machine learning techniques (e.g., SVM[6]) have been combined with relevance feedback mechanism to estimate the user's retrieval concept.

Note that in most cases, the example images provided by users only characterize partial information of users' true search targets, which is a typical localized CBIR (LCBIR) task. However, most existing relevance feedback based retrieval approaches used the global low-level visual features to represent an image, which could not deal with the LCBIR issue. Not much attention has been paid to developing approaches to solve this problem. One effort in this direction is proposed by Rahmani et al. [2] who utilized the multiinstance learning algorithm to learn the target concept from the training data provided by the user's feedback. Despite the retrieval performance being satisfactory, the drawback lies in that such LCBIR approach is a purely top-down learning manner due to the fact that the region-of-interest is learnt by the query set provided by the user, where the data-driven bottom-up mechanism is ignored. However in most cases, the salient parts of an image are usually consistent with user's retrieval concept. Furthermore, the multi-instance learning used in [2] is very time-consuming which does not fit the real-time query requirement.

Being aware of this, we try to estimate the user's retrieval concept to perform the LCBIR task via a combination of both bottom-up and top-down manner. As to the bottomup manner, the computational visual attention model can well locate the visual salient part of a given image purely without any user's interaction. Under most circumstances, such extracted salient part is consistent with user's interest. In contrast, the relevance feedback mechanism which needs users to provide both positive and negative samples to learn the target concept, is also widely used for the CBIR task as a typical top-down manner. Instead of the multi-instance learning algorithm proposed in [2], we try to use the graph learning framework to learn the user's retrieval concept. We argue that, in cases where region significance is considered in the graph learning based LCBIR algorithm, both human perception and internal image manifold structure can be well encoded. Since the manifold ranking algorithm can rank the data points along their underlying manifold by analyzing their relationship in Euclidean space, it can be naturally 
well applied to measure the relevance between images denoted by the high-dimension features. The proposed method is evaluated on the well-known SIVAL datasets [2] and we show that it outperforms the state-of-the-art competitive approaches.

The organization of the paper is as follows. The detail of visual attention model based image analysis is proposed in Sect. 2. Then, Sect. 3 describes the proposed graph learning algorithm where region saliency factor is encoded. Experimental results are reported in Sect. 4. Finally, conclusions will be presented in Sect. 5 .

\section{Visual Attention Based Image Analysis}

As aforementioned, under most circumstances, the salient parts of an image are usually consistent with user's query concept. So we firstly introduce an improved saliency map generation algorithm. Inspired by [3], [10], we simply define the multi-scale contrast features as a linear combination of contrasts in the Gaussian image pyramid. For a given color image $I$ in the original RGB color space, suppose $p_{x}^{I}$ is a pixel in a given image $I, \theta_{p_{x}^{I}}$ is a size of $3 \times 3$ window centered at $p_{x}^{I}$ for the computation of contrast. Here we consider color, intensity, and orientation features. The saliency value for the pixel $p_{x}^{I}$ can be calculated as:

$$
S P\left(p_{x}^{I}\right)=\sum_{l=1}^{L} \sum_{p_{y}^{I} \in \theta_{p_{x}^{I}}}\left(\gamma_{C I} S_{C I}^{l}\left(p_{x}^{I}, p_{y}^{I}\right)+\gamma_{O} S_{O}^{l}\left(p_{x}^{I}, p_{y}^{I}\right)\right)
$$

where $p_{y}^{I}$ is the pixel belonging to $\theta_{p_{x}^{I}} . S_{C I}\left(p_{x}^{I}, p_{y}^{I}\right)$ and $S_{O}\left(p_{x}^{I}, p_{y}^{I}\right)$ denote the color-intensity contrast and orientation contrast between $p_{x}^{I}$ and $p_{y}^{I} \cdot \gamma_{C I}$ and $\gamma_{O}$ are the weighting coefficients and set to 1 for simplicity. $l$ denotes the level image in the pyramid and the number of pyramid levels $L$ is 3 . After the linear combination is finished, we use a Gaussian filter with the standard deviation $\sigma=1$ to remove the noise points. Figure 1 gives some examples of our proposed computational model to build saliency map.

We adopted the well-known JSEG [9] method to segment the image into homogeneous regions. For each segmented region $r_{i}^{I}$ in the image $I$, the region average saliency value $S R\left(r_{i}^{I}\right)$ can be calculated as follows:

$$
S R\left(r_{i}^{I}\right)=\sum_{p_{x}^{I} \in r_{i}^{I}} S P\left(p_{x}^{I}\right) \mid \operatorname{Area}\left(r_{i}^{I}\right)
$$

where $S P\left(p_{x}^{I}\right)$ and $S P\left(r_{i}^{l}\right)$ denote the saliency values of pixel

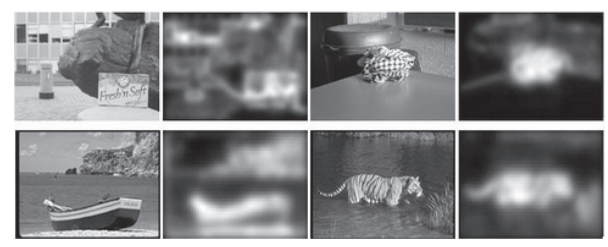

Fig. 1 Examples of saliency map generated by the proposed method.
$p_{x}^{I}$ and region $r_{i}^{l}$, respectively.

\section{An Improved Graph Learning Algorithm}

In this section, we want to provide some details concerning about how to perform the relevance feedback on the region level. In a typical relevance feedback scheme, both positive and negative images are taken into account and the relevance feedback process is considered a classification problem. Both positive and negative images provided by the user can be treated as training examples and a classifier could be trained. Then, all the data set can be classified into relevant and irrelevant groups. Support vector machine (SVM) based feedback approach has been widely applied in CBIR. However, existing approaches require the use of fixed-length global-level visual feature representation, which does not fit the LCBIR task. The key issue of LCBIR is to learn the target concept given the positive and negative images provided by the user's feedback. Being aware of this, we propose an improved graph learning framework to resolve such issue.

Graph-based methods [4] have achieved much success in the field of image retrieval [5] and automatic image annotation [8], which formulated the image retrieval task as a semi-supervised learning problem. Specifically, [5] take the user's query image as a labeled data and the images in the dataset as the unlabeled data, then the labels are propagated from the labeled data to the unlabeled data along their underlying manifold by analyzing their relationship in Euclidean space.

In contrast to the existing graph-based algorithm which aims to propagate the labels from labeled to unlabeled samples, we utilize the algorithm to search for the target concept extraction. The main idea behind such setting lies in that, all the positive images may have visually similar patterns while all the negative images are out of users' query target. Note that in the traditional graph-based image retrieval algorithms [5], the algorithm takes each image in the dataset as a vertex to form a weighted graph. In contrast, in our graph learning algorithm, segmented regions from the user's feedback image set are considered as the vertexes to form the affinity matrix. The detail of the proposed graph learning algorithm is given below.

Specifically, the user formulates an initial query via a simple global-level visual feature matching strategy (This step does not need a sophisticated matching strategy). Without loss of generality, assume a set of images given by the user's initial feedback is denoted by $€=L^{+} \cup L^{-}$, where $L^{+}=\left\{I_{1}^{+}, I_{2}^{+}, \ldots I_{|P|}^{+}\right\}$and $L^{-}=\left\{I_{1}^{-}, I_{2}^{-}, \ldots I_{|N|}^{-}\right\}$denote the positive and negative images, respectively. $|P|$ and $|N|$ denote the number of positive and negative samples. $R=\left\{r_{1}, r_{2}, \ldots r_{|R|}\right\}$ denotes all the regions derived from $L$ and $|R|$ is the region number in $L$. Take each region $r_{i} \in R$ to denote a node in the affinity matrix $W^{|R| \times|R|}$, each element $w_{i j}$ is the visual similarity between region $r_{i}$ and $r_{j}$. The definition of $W^{|R| \times|R|}$ will be explained later. A vector $Y \in \mathbf{R}^{|R| \times 1}$ is defined to measure the relevant degree that each region $r_{i} \in R$ belongs to the user's query target concept. The initial 
vector is set as $Y=\left[y_{1}, y_{2}, \cdots, y_{|R|}\right]^{T}$. Since there does not exist any query target concept in the negative images, so for each region in the negative images, the initial label equals to -1 ; while for each region in the positive images, since not all the regions in the positive images fit for the query target concept, we cannot simply assign each region with label +1 . Instead, we assume that regions in the positive images with more salient manners may have higher probability to be the query target concept. So we assign the initial labels of regions equal to the corresponding normalized saliency value. Based on the above assumption, the initial label vector $Y=\left[y_{1}, y_{2}, \cdots, y_{|R|}\right]^{T}$ is defined as:

$$
y_{i}=\left\{\begin{array}{ll}
S R\left(r_{i}\right), & r_{i} \in I^{+} \\
-1, & r_{i} \in I^{-}
\end{array} \quad i=1, \ldots,|R|\right.
$$

We take the above affinity matrix $W^{|R| \times|R|}$ and the initial label vector $Y$ in the graph learning algorithm. So the procedure of the proposed algorithm can be described below.

1. Sort the pair-wise distances among points in ascending order. Repeat connecting the two points with an edge according to the order until a connected graph is obtained.

2. Form the affinity matrix $W$ defined by $W_{i j}=$ $\exp \left[-d^{2}\left(r_{i}, r_{j}\right) / 2 \sigma^{2}\right]$ if there is an edge linking $r_{i}$ and $r_{j}$. Let $W_{i i}=0$.

3. Symmetrically normalize $W$ by $S=D^{-1 / 2} W D^{-1 / 2}$ in which $D$ is the diagonal matrix with $(i, i)$-element equal to the sum of the $i$ th row of $W$.

4. Iterate $f(t+1)=\alpha S f(t)+(1-\alpha) Y$ until convergence, where $\alpha$ is a parameter in $[0,1)$ and $f(0)=Y$.

5 . Let $f^{*}$ denote the limit of the sequence $\{f(t)\}$. Rank each point $r_{i}$ according to its ranking probability values $f_{i}^{*}$.

Note that the traditional graph-based algorithm aims to propagate the class labels from the labeled data to unlabeled data, while in our cases, the key point is the positive images in the feature space are likely to form a compact cluster; in contrast, the negative images can be varied in the feature space. Such assumption can well fit the current relevance feedback strategy. So after the iterations, regions in the positive images which can well represent the user's query target will get higher label values. Then we use the threshold $y_{\text {thres }}$ to select regions to form the query target concept set, i.e., regions with probability values higher than $y_{\text {thres }}$ are selected as query target concept. Similar to [7], the threshold $y_{\text {thres }}$ is defined as:

$$
y_{\text {thres }}=\max \left\{y^{*} \mid \forall I_{i}^{+} \in L^{+}, \exists r_{i} \in I_{i}^{+} \text {, s.t. } y_{i}^{*} \geq y^{*}\right\}
$$

Given the target concept set $R^{*}=\left\{r_{1}^{*}, r_{2}^{*}, \ldots, r_{\left|R^{*}\right|}^{*}\right\}$ as the new query set, the retrieval system then return the images which are visually similar to the set. Assume the image set in the database $U=\left\{I_{1}, I_{2}, \ldots, I_{|U|}\right\}, I_{i}=\left\{p_{1}^{I_{i}}, p_{2}^{I_{i}}, \ldots, p_{\left|I_{i}\right|}^{I_{i}}\right\}$. We adopt the minimal Hausdorff distance to determine the distance between $I_{i} \in U$ and each target region in the target concept set $r_{j}^{*} \in R^{*}$, which is defined as:

$$
d\left(r_{j}^{*}, I_{i}\right)=\min _{k=1, \ldots,\left|I_{i}\right|} \operatorname{dist}\left(r_{j}^{*}, p_{k}^{I_{i}}\right)
$$

where $\operatorname{dist}(\mathrm{)}$ is the Euclidean distance of two regions' visual feature vectors. So the probability that image $I_{i} \in U$ fits for the users' query concept is as follows:

$$
l_{I_{i}}=\frac{1}{\left|R^{*}\right|} \sum_{j=1}^{\left|R^{*}\right|} e^{-d\left(r_{j}^{*}, I_{i}\right)^{2}}
$$

Then the retrieval results can be returned to the user according to the probability value in order. If the user is still unsatisfied with the results, then the similar strategy can be implemented until the user is satisfied.

\section{Experimental Results}

We conduct several experiments to demonstrate the effectiveness of the proposed method. We show the retrieval performance of the proposed algorithm as compared with [2] using the SIVAL benchmark. The SIVAL (Spatially Independent, Variable Area, and Lighting) benchmark consists of 25 different objects, and 10 scenes. There are 6 different images taken for each object-scene pair, and a total of 1500 images in the database. SIVAL emphasizes the task of localized CBIR through nearly identical scenes which only vary by the localized target objects. We compare the performance of the proposed method and ACCIO! [2] for all 25 object categories of SIVAL.

We first give some examples in Fig. 2 to evaluate the target concept set extraction performance as compared with original manifold learning without taking regional saliency into consideration, i.e., we simply assign each region in the positive image set with label +1 instead of its corresponding salient value. Here 4 positive from the "fabricsoftenerbox" category and 4 negative images are randomly selected in SIVAL dataset to learn the target concept set. From the figure, we can cleary see that results with the proposed method on the third column apparently outperforms the results on the fourth column with the original manifold learning method.

In addition, consistent with existing works, we use AUC as the performance measure. In the image retrieval, the area under the receiver-operating characteristic (ROC) curve (AUC) is a good measure of the retrieval performance.

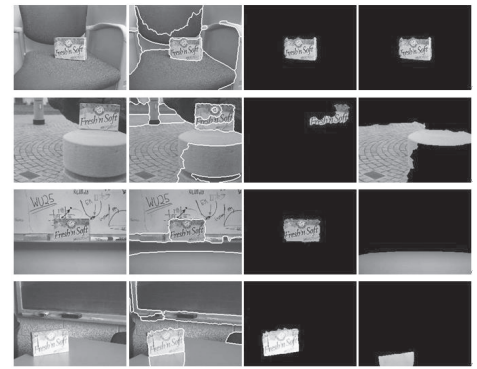

Fig. 2 Performance comparison of target concept detection. From left to right: the original images, the segmented images, results with taking region saliency into account, results without taking region saliency into account, respectively. 


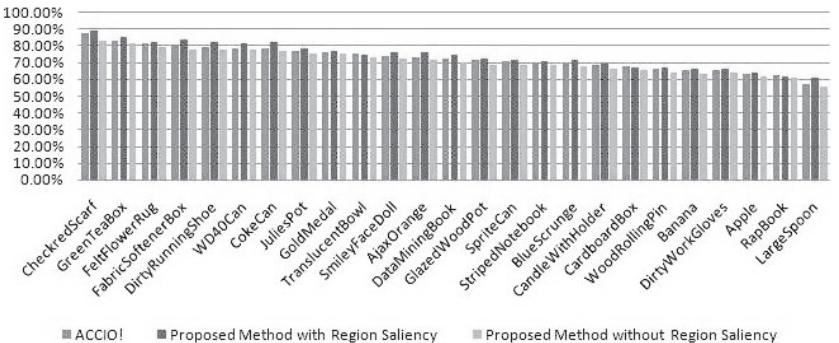

Fig. 3 Performance comparision of different algorithms on the SIVAL dataset.

The ROC curve plots the true positive rate as a function of the false positive rate. For ACCIO!, 8 random positive and 8 random negative examples from the image dataset are placed in the query image set, which are used for target concept detection under Multi-Instance Learning (MIL) framework. The retrieval performance is presented in Fig. 3. It shows that the proposed method outperforms the original manifold-ranking based method, and can achieve similar retrieval accuracy as compared with ACCIO!. However, the computational cost of our method is apparently lower than ACCIO! since ACCIO! needed the iterative computing under diverse density framework to acquire the target concept.

\section{Conclusion}

In this paper, we have proposed a novel saliency-based graph learning framework to solve the localized contentbased image retrieval (LCBIR) task. Firstly, the visual attention model is employed to measure the regional saliency of each image in the feedback image set provided by the user. Secondly, the regions in the image set are constructed to form an affinity matrix and a novel propagation energy function is defined which takes both low-level visual features and regional significance into consideration. After the iteration, regions in the positive images with high confidence scores are selected as the candidate query set to conduct the nextround retrieval task until the retrieval results are satisfac- tory. Experimental results conducted on the SIVAL datasets demonstrate the effectiveness of the proposed approach. In future work, we plan to extend the framework in the field of image annotation and object recognition.

\section{Acknowledgements}

This work was supported by the Fundamental Research Funds for the Central Universities (2009JBM024) and the National Nature Science Foundation of China (No.60972145, 61033013).

\section{References}

[1] R. Datta, D. Joshi, J. Li, and J.Z. Wang, "Image retrieval: Ideas, influences, and trends of the new age," ACM Comput. Surv., vol.40, no.2, pp.1-60, 2008.

[2] R. Rahmani, S.A. Goldman, H. Zhang, J. Krettek, and J. Fritts, "Localized content based image retrieval," Proc. ACM Int. Conf. on Multimedia Information Retrieval (ACM MIR05), pp.227-236, Singapore, Nov. 2005.

[3] L. Itti, C. Koch, and E. Niebur, "A model of saliency-based visual attention for rapid scene analysis," IEEE Trans. Pattern Anal. Mach. Intell., vol.20, no.11, pp.1254-1259, 1998.

[4] D. Zhou, O. Bousquet, T.N. Lal, J. Weston, and B. Scholkopf, "Learning with local and global consistency," Proc. NIPS'03, 2003.

[5] J. He, M. Li, H.J. Zhang, H.H. Tong, and C. Zhang, "Generalized manifold-ranking based image retrieval," IEEE Trans. Image Process., vol.15, no.10, pp.3170-3177, 2006.

[6] F. Jing, M. Li, H.J. Zhang, and B. Zhang, "An efficient and effective region-based image retrieval framework," IEEE Trans. Image Process., vol.13, no.5, pp.699-709, 2004.

[7] J. Tang, X.S. Hua, G.J. Qi, and X. Wu, "Typicality ranking via semi-supervised multiple-instance learning," Proc. ACM Int. Conf. on Multimedia (ACM Multimedia'07), pp.297-300, Augsburg, Germany, Sept. 2007.

[8] J. Liu, M. Li, Q. Liu, H. Lu, and S. Ma, "Image annotation via graph learning," Pattern Recognit., vol.42, no.2, pp.218-228, 2009.

[9] Y. Deng and B.S. Manjunath, "Unsupervised segmentation of colortexture regions in images and video," IEEE Trans. Pattern Anal. Mach. Learn., vol.23, no.8, pp.800-810. 2001.

[10] Y. Sun and R. Fisher, "Object-based visual attention for computer vision,” Artif. Intell., vol.146, pp.77-123, 2003. 